\section{$\underset{\text { hommes }}{\text { \& migrations }}$}

\section{Hommes \& migrations}

Revue française de référence sur les dynamiques

migratoires

1303 | 2013

Diasporas marocaines

\title{
Le Maroc, un carrefour migratoire pour les circulations euro-africaines?
}

\author{
Mehdi Alioua
}

\section{(2) OpenEdition}

\section{Journals}

\section{Édition électronique}

URL : http://journals.openedition.org/hommesmigrations/2572

DOI : 10.4000/hommesmigrations.2572

ISSN : 2262-3353

\section{Éditeur}

Musée national de l'histoire de l'immigration

\section{Édition imprimée}

Date de publication : 1 juillet 2013

Pagination : 139-145

ISBN : 978-2-919040-23-0

ISSN : $1142-852 X$

\section{Référence électronique}

Mehdi Alioua, «Le Maroc, un carrefour migratoire pour les circulations euro-africaines ? ", Hommes \& migrations [En ligne], 1303 | 2013, mis en ligne le 31 décembre 2015, consulté le 03 mai 2019. URL : http://journals.openedition.org/hommesmigrations/2572; DOI : 10.4000/hommesmigrations.2572 


\section{UN CARREFOUR MIGRATOIRE POUR LES CIRCULATIONS EURO-AFRICAINES?}

par MEHDI ALIOUA, professeur assistant en sociologie à l'Institut d'études politiques et juridiques de l'université internationale de Rabat, et chercheur associé au Centre Jacques-Berque.

Le Maroc est devenu une étape clé pour les migrants subsahariens qui cherchent à quitter le continent africain. La durée de l'étape n'étant pas fixée à l'avance, ils ont besoin de se loger, de travailler et d'obtenir toutes sortes d'informations nécessaires à la poursuite de leur aventure. Dans les grandes villes du royaume chérifien où ils font halte, les migrants trouvent des relais sociaux essentiels auprès des migrants installés précédemment ou de la population locale. Ainsi se met en place un tissu économique fondé sur la débrouille et la circulation. Une mondialisation par le bas.

\section{Des dizaines de milliers de personnes aux origines multiples et aux choix de destinations variés}

La migration transnationale par étapes et par contournementdesfrontières-outransmigration ${ }^{1}-$ des Africains subsahariens en route vers l'Europe est une forme migratoire où déterritorialisation, reterritorialisation et redéterritorialisation cadencent le temps et l'espace. Pour ces migrants, souvent sans visa, l'espace migratoire transnational qu'ils s'aménagent n'est pas lisse, leur migration se déroule durant plusieurs années et dans plusieurs pays qui n'ont pas prévu leur venue et leur installation. La dimension spatio-temporelle ${ }^{2}$ doit donc être impérativement replacée dans ce contexte où les trajectoires migratoires sont rythmées par des étapes au cours desquelles les migrants se réorganisent, le temps de passer les frontières qui s'érigent devant eux. Ils doivent à chaque étape de leur parcours s'insérer dans les lieux où ils sont, y vivre, y habiter, y travailler, y commercer, s'y soigner, parfois même y défendre leurs droits ${ }^{3}$ avant d'essayer de passer à l'étape suivante. Au niveau quantitatif, il ne s'agit que de quelques dizaines de milliers de personnes aux origines multiples et aux choix de destinations variés, rien qui devrait mobiliser autant l'opinion publique des pays européens et maghrébins, ni faire l'objet d'autant d'attention de la part des services de sécurité de tous 
ces pays, ni d'autant de lois et décrets qui les visent directement ${ }^{4}$. Pourtant, ce mouvement migratoire a un impact très fort dans la région, notamment au Maroc.

Je travaille depuis 2002 sur cette forme migratoire en saisissant les mouvements de ces populations migrantes d'Afrique noire, qui s'incarnent dans des espaces sociaux et des territoires transnationaux, perpétuant les tensions de leurs mobilités et de leurs ancrages dans les pays d'Afrique de l'Ouest, du Maghreb et de l'Europe en général, et au Maroc en particulier. Notre étude des réseaux transnationaux qui les supportent pose la question de la conception de configurations spatiales non limitées, produites par ces populations en mouvement, mais dans un contexte géopolitique où les frontières ne sont pas aussi poreuses que le suppose le terme de "transnational".

Pour y répondre, la notion d'étape, appréhendée à la fois comme lieu d'observation, cadrage méthodologique et outil analytique, me paraît bien plus pertinente que celle de "transit", notamment parce que, pour comprendre cette migration et surtout les conséquences qu'elle provoque sur les territoires investis, il faut au

Toujours étrangers dans les sociétés minimum un temps et un lieu d'observation.

qu'ils traversent, les migrants ont dû réorganiser De plus, il ne s'agit pas seulement de rendre compte de l'autonomie sociale de ces migrants et de leur capacité à contourner les frontières et à renégocier localement certaines règles du jeu des "sédentaires", savoir-faire qui participent à la construction d'espaces transnationaux dans lesquels ils réussissent tant bien que mal à circuler. Il s'agit de mettre au jour les difficultés à passer toutes ces "épreuves", toutes ces "barrières" qui ne se situent pas qu'aux seules limites géographiques des États-nations, mais aussi à l'intérieur, le plus souvent dans leurs marges, comme en périphérie des grandes villes. Car, pour pouvoir circuler, il leur faut des relais et des haltes où ils puissent se relocaliser et trouver toutes les informations, les connexions et les ressources (économiques, sociales et symboliques) nécessaires à la survie et au prochain passage souhaité

\section{Le Maroc, pays étape plutôt que pays de transit}

Dans le cas des Africains subsahariens au Maroc désirant passer en Europe mais se sentant bloqués, on pourrait accorder la primauté de l'analyse sociologique aux seuls projets migratoires originels. Il y aurait la tentation de réduire ce type de migration au seul "transit" et donc de parler du Maroc en tant que "pays de transit", de ce mouvement comme une "migration de transit" et de ces personnes comme des "migrants de transit". Toutefois, ces terminologies sont à la fois insatisfaisantes sur le plan de la compréhension sociologique, mais également suffisamment ambiguës politiquement pour induire le chercheur en erreur.

La notion de "transit" correspond à la place que ce mot occupe aujourd'hui au sein de nos sociétés, tant comme objet de discussion entre "experts" que comme enjeu de débats publics ${ }^{6}$. En Europe, par exemple, le terme de "pays de transit" est curieusement dédié dans le sens commun aux pays limitrophes de l'Union européenne, accentuant encore plus le présupposé de l'extériorité de ces pays et l'idée qu'une "frontière naturelle" sépare l'Union européenne du reste du monde. Il faut se rappeler pourtant que des pays comme l'Espagne ou l'Italie étaient considérés comme des "pays de transit" dans les années 1990, avant de devenir d'importants pays d'immigration. De plus, d'anciens pays dits de "transit" tels que Chypre ou Malte ont 
Deux migrants soudanais marchent dans la forêt en attendant le bon moment pour franchir la frontière entre le Maroc et Mellila, dans la province de Nador, le 20 octobre 2005 (c) AFP / PHoto by MARCo DI LAURo/GetTy Images

changé de statut du seul fait de leur entrée dans l'Union, devenant "premiers pays d'entrée dans l'Union européenne", alors que nous savons bien qu'une majorité de migrants ne veulent pas s'installer dans ces pays mais tenter d'entrer dans l'espace Schengen!

De plus, l'idée même de "transit" est extrêmement restrictive du point de vue spatio-temporel, évoquant plutôt le temps privilégié de la flânerie dans le hub d'un aéroport international où, en attendant son prochain vol, l'on erre, en regardant les vitrines des magasins détaxés. Nous sommes loin, très loin, de ce que vivent ces migrants qui traversent l'Afrique, le plus souvent sans autorisation administrative, à la recherche d'un lieu qui pourra leur permettre de réaliser leur projet ou de trou- ver refuge. C'est en partie pour cela que la notion d'étape rend mieux compte de la complexité de ces parcours migratoires en replaçant la dimension spatio-temporelle, qui ne se réduit pas à l'attente dans un non-lieu ${ }^{7}$ avec un minimum d'interactions avant de passer dans un autre.

L'étape, c'est un temps bien plus long, bien plus complexe, durant lequel les imprégnations, les interactions sociales, les rapports de domination ainsi que les conflits qui en résultent sont suffisamment importants pour transformer les acteurs du fait migratoire, autant que ceux qui les voient passer et s'installer. Les migrants dont nous parlons, quasi nomades, toujours étrangers dans les sociétés qu'ils traversent, ont dû réorganiser collectivement leur errance. Cela est encore plus parlant pour ceux qui ont prolongé leur séjour au Maroc, par 
choix ou par défaut. Si certains avaient déjà ce projet en tête, ou alors avaient anticipé l'effet de temporalité inhérent à cette forme migratoire, d'autres, se retrouvant bloqués dans ce pays, tentent de s'y réaménager une vie. Mais tous n'y parviennent pas : pour réussir à vivre - où devrions-nous écrire "survivre" ? -, ils doivent aussi s'insérer dans le tissu social local. Pour cela, ils ont établi dans ce pays des espaces d'installation élaCe sont essentiellement borés comme des relais miles grandes villes du Maroc gratoires à partir de relais qui deviennent des relais migratoires, dont l'importance ne cesse de croître sous l'afflux constant des migrants. sociaux. Il est aisé de concevoir que si ces migrants subsahariens, quels que soient les statuts qu'on leur impose ou les origines dont ils se revendiquent, appréhendent le Maroc comme une étape sur la route, c'est qu'ils pensent y trouver des relais sociaux qui leur permettront de s'y introduire et d'y trouver des moyens de subsistance. De façon générale, si de nouveaux migrants subsahariens ne cessent d'arriver et de circuler au Maroc, c'est bien parce qu'ils y trouvent des personnes-ressources qui leur indiquent comment s'insérer afin d'y survivre jusqu'au prochain départ. Ils ont alors organisé collectivement dans le même temps la solidarité, de manière ethnico-communautaire, et la coopération, en dépassant leurs appartenances nationales et ethniques, entre migrants subsahariens mais aussi avec certains Marocains.

\section{L'émergence des relais migratoires}

Ce sont essentiellement les grandes villes du Maroc qui deviennent des relais migratoires, dont l'importance ne cesse de croître sous l'afflux constant des migrants. Or ces lieux étaient déjà des étapes pour les migrants marocains qui faisaient leur pre- mière entrée en ville en s'installant dans des quartiers populaires périphériques. Par exemple, des quartiers comme ceux de la commune de Yacoub El Mansour à Rabat ou comme Oulfa à Casablanca étaient déjà imprégnés par toutes les relations sociales tissées par les migrants de l'intérieur ${ }^{8}$. Nous savons aussi que les villes de Tanger, Rabat ou Casablanca sont, depuis les années $1980^{\circ}$, des relais migratoires pour les Marocains candidats à l'émigration, avant de le devenir aussi pour les migrants subsahariens.

Alors qu'avant on émigrait depuis la campagne et les petits douars, c'est depuis ces trois villes principales que l'on migre aujourd'hui vers l'international : plus du tiers des nouveaux émigrants y ont vécu au moins un an avant leur départ. De plus, ces trois villes deviennent aussi des lieux de retour pour certains migrants marocains vivant à l'étranger qui préfèrent souvent revenir en vacances ou se réinstaller dans une ville proche de ce qu'ils ont connu à létranger, plutôt que dans leur douar d'origine. Et elles sont aujourd'hui les villes principales d'installation pour les migrants originaires des pays d'Afrique noire, pour ceux qui veulent passer en Europe comme pour ceux qui désirent s'établir plus durablement. Les relais migratoires dont nous parlons émergent des quartiers populaires, souvent périphériques, de ces trois grands centres urbains où les migrants de l'intérieur et aujourd'hui d'Afrique noire, souvent en situation de déshérence lorsqu'ils font leur entrée en ville, ont dû se reconstituer une "vie sociale". Ils ont aménagé collectivement ces espaces dans lesquels ils s'installent, circulent, et qu'ils finissent par "habiter". Bref, ils doivent "se débrouiller ${ }^{10 "}$ pour y vivre. Ces lieux, connectés au monde grâce aux circulations migratoires, deviennent ainsi les supports d'une mondialisation par le bas.

Par exemple, beaucoup d'habitants de ces quartiers populaires survivent grâce aux mandats envoyés

8. Mehdi Alioua, "La migration transnationale des Africains subsahariens au Maghreb. L'exemple de l'étape marocaine”, in Ali Bensaâd (dir.), Maghreb Machrek, $n^{\circ} 185$, "Marges et mondialisation. Les migrations transsahariennes", 2005, pp. 37-58. 9. Dans les années 1980, un tournant s'opère dans le dispositif migratoire marocain : ces grandes villes deviennent des espaces rebonds pour les migrants de l'intérieur ou pour leurs enfants qui y ont grandi, qui n'y trouvent plus leur place et qui se mettent à élaborer des projets d'émigration internationale. 10. Olga L. Gonzalez, “La 'débrouille' : migrants andins et accès aux droits en France”, doctorat en sociologie sous la direction de Michel Wieviorka, Paris, Cadis, EHESS, 2007. 
par un proche qui a migré à l'étranger ; beaucoup de petites maisons y ont été construites grâce à ces transferts, leur permettant de passer de la tôle au dur, d'un rez-de-chaussée à une maison à étage, transformant le paysage urbain. Les difficultés d'accès au financement banquier par crédit pour les habitants de ces quartiers populaires font que l'autofinancement représente $80 \%$ des logements réalisés ${ }^{11}$. Une bonne part de cet autofinancement provient des revenus transférés des migrants marocains vivant à l'étranger, puis est remboursée (voire rentabilisée) par la location d'une partie du logement. Généralement, ces nouveaux propriétaires construisent étage par étage, et ils financent leur investissement et leurs travaux au fur et à mesure, grâce à des locations. La plupart de ces locataires sont de nouveaux migrants de l'intérieur venus grossir les rangs des travailleurs précaires, en laissant derrière eux leur famille à qui ils envoient la plus grande partie de leur modeste salaire. Avec le temps, certains d'entre eux décident de s'installer à long terme et changent de mode d'habitation, devenant parfois eux-mêmes propriétaires, et/ou élaborent des projets d'émigration internationale, mais ils sont aussitôt remplacés par de nouveaux venus.

Depuis les années 2000, les migrants subsahariens représentent aussi une part très importante et sous-estimée de ces locataires. Ces nouveaux venus participent à cette économie de la débrouille, cet "entre-pauvres", et permettent à certaines familles marocaines de s'assurer un petit revenu, voire de devenir propriétaires. Même si beaucoup de ces migrants subsahariens finissent par quitter le Maroc, d'autres s'installent et de nouveaux arrivent, remplaçant les anciens dans une sorte de noria. Cette clientèle ne se tarit pas. Au contraire. Or pour pouvoir louer une chambre, pour pouvoir acheter à manger et de quoi se soigner, s'habiller, etc., il leur faut des revenus : à l'instar de nombreux Marocains qui vivent dans ces quartiers, ils sont journaliers, ouvriers dans le bâtiment, la restauration, la briqueterie, etc. ; ils sont parfois men- diants ou chiffonniers. Beaucoup sont marchands ambulants ou "guides" pour marchands, sorte d'intermédiaires et d'interprètes facilitant la rencontre commerciale, voire la confiance. Si on ajoute à cela le fait qu'ils reçoivent souvent de l'argent envoyé par un proche, généralement immigré en Europe, non seulement ils ressemblent par ces stratégies de débrouillardise à leurs voisins marocains, mais ils s'inscrivent ainsi dans une économie de la circulation. En grossissant les files d'attente de la Western Union - qui fleurissent dans ces quartiers, pourtant pauvres en apparence - où ils viennent chercher le mandat envoyé par un proche vivant en Europe, tous les habitants de ces quartiers, nationaux Même si beaucoup ou étrangers, ont les mêmes de ces migrants subsahariens stratégies et les mêmes finissent par quitter le Maroc, pratiques de solidarité : la d'autres s'installent et de dispersion et la circulation nouveaux arrivent, remplaçant dans l'espace. Les migrants les anciens dans une sorte subsahariens se sont ainsi de noria. complètement insérés dans le tissu urbain de ces villes, les transformant même par leur présence. En venant s'installer dans ces quartiers, ils greffent leurs propres circulations sur celles instituées localement et historiquement.

\section{Une économie de la circulation et de la débrouille}

Ces migrants subsahariens participent à l'économie locale qui échappe, partiellement ou entièrement, aux contrôles de l'État marocain. Il ne s'agit pas vraiment d'une économie "souterraine", ni non plus d'une économie "illicite" ou alors "amorale"; ni, encore moins, d'une économie "informelle". C'est une économie de la circulation et de la débrouille qui est très formalisée socialement, car les acteurs doivent se faire confiance en dehors de la protection juridique et administrative des autorités étatiques. Les migrants subsahariens ne signent pas de bail, ne déposent pas de caution. Ils ne donnent 
pas de préavis lorsqu'ils quittent leur logement ni ne font d'état des lieux. Les propriétaires ne paient pas d'impôt sur ces revenus ni ne demandent l'autorisation administrative d'élever d'un étage leur maisonnette. Tout se fait dans une confiance mutuelle, ce qui n'est pas sans conflits

Cette économie

de la circulation et de la débrouille est visible et fait dispositif dans l'étape urbaine. Elle peut parfois prétendre à organiser des créneaux économiques particuliers, voire des pans entiers de l'économie

commerciale dans son interface locale-globale, ou du moins, transfrontalière. ni sans exploitation ou rapports de pouvoir. Mais, de la même manière que les migrants qui ont traversé le Sahara ont bien été obligés de faire confiance à leur "passeur" auquel leur sort était lié le temps du convoyage "clandestin", ils doivent faire confiance à leurs bailleurs. Et ces derniers qui souvent vivent avec ces migrants doivent eux aussi avoir con-

fiance dans le paiement du loyer, car les retards sont courants. Tous ces acteurs négocient sans cesse, étant toujours en relation de face-à-face. Nous sommes bien loin de la dépersonnalisation presque totale du virement bancaire mensuel à une agence de location qui vire ensuite la somme due au propriétaire qui ne connaît même pas son ou ses locataires, comme cela se produit souvent en Europe. L'économie de la circulation et de la débrouille dont je parle - qui est proche de la forme de l'économie de bazar proposée par Geertz ${ }^{12}$ - ne peut reposer que sur un espace social qui fonctionnait déjà de cette manière. Cette configuration économique, caractérisée d'abord par la forme interpersonnelle des échanges - le "face to face" -, ensuite par l'importance de la parole donnée, concerne en premier lieu les Marocains qui ont immigré dans ces grandes villes. C'est une économie de petits boulots et de petits commerces qui mobilise très peu de moyens matériels et financiers. On pourrait aussi la nommer "économie de subsistance", mais je préfère garder le terme de "débrouille" qui suggère une certaine créativité et la capacité d'ac- tion des individus qui la pratiquent. Elle se fait au grand jour, contrairement à l'économie souterraine qui nécessite un minimum de discrétion, voire, dans le cas d'une commercialisation de produits illicites, d'invisibilité. Cette économie de la circulation et de la débrouille est visible et fait dispositif dans l'étape urbaine. Elle peut parfois prétendre à organiser des créneaux économiques particuliers, même des pans entiers de l'économie commerciale dans son interface locale-globale, ou du moins, transfrontalière ${ }^{13}$. Ici, le contexte est celui de la "clandestinité" et de la nécessité de survivre : les migrants subsahariens sont entrés au Maroc ou y ont prolongé leur séjour sans l'autorisation de l'État marocain. Il y a pourtant une similitude dans certains aspects du mode de vie de ces populations et dans leurs rapports aux normes sociales édictées par des pouvoirs centraux dont elles se méfient. Évidemment, cela n'est exempt ni de rapports de force ni de conflits, mais comme en plus de la proximité sociale il y a une complémentarité économique, ces espaces continuent d'accueillir de nouveaux venus en les intégrant à la ville. Surtout qu'un tel environnement social, reposant sur un espace de confluence voulu par la politique marocaine qui tente de faire du pays un carrefour commercial, facilite l'initiative économique. Certes, c'est plutôt une économie de la débrouille qui émerge, mais elle permet à un grand nombre de personnes de vivre, parfois même de prospérer, offrant une alternative aux voix classiques de l'intégration économique, ici au Maroc, comme ailleurs en Afrique et en Europe ${ }^{14}$.

\section{Le Maroc, un carrefour euro-africain}

Dans le même temps, la politique d'ouverture du Maroc à la mondialisation libérale s'accélère. Avec la politique volontariste du chef de l'État, le roi Mohamed VI, cette nation espère se dévelop- 
per économiquement en devenant un carrefour pour l'espace euro-africain. Pour cela, il s'agit, d'une part, de développer les infrastructures, particulièrement celles des voies de circulation et de télécommunication. Et, d'autre part, de faire évoluer les institutions, les règles et les normes vers cette nouvelle politique économique : adhésion à des zones de libre échange et de libre circulation des marchandises avec suppression (ou diminution) des barrières non tarifaires, création de zones franches, diminution du coût des transactions, convertibilité du compte de capital, etc. Au-delà de ces recettes économiques, la stratégie du Maroc est d'utiliser sa position géographique en jouant le rôle de carrefour, c'est-à-dire devenir un hub et une zone off-shore pour reprendre les termes de la mondialisation libérale. Une grande partie de ce chantier est donc dédiée aux circulations, à leurs différentes formes, à leurs convergences et à leurs interconnexions : import-export, transbordement, assemblage pour export, fret, réexportation, transport de populations, lignes à grande vitesse (avec le TGV français pour 2015), autoroutes, tourisme, etc.

Mais, si en s'ouvrant de la sorte, le Maroc se développe d'un côté - il connaît depuis 2003 une forte croissance du PIB -, d'un autre, les inégalités interpersonnelles et entre les territoires s'accroissent : il y a des zones de pauvreté qui n'en profitent pas suffisamment.

Pour autant, certains Marocains ne restent pas à attendre que le développement vienne à eux : ils prennent des initiatives et tentent de pallier leur exclusion. La migration, interne ou internationale, le contournement de certaines frontières ou règles administratives, l'économie souterraine, la débouille, font partie de ces initiatives. Et, paradoxalement, grâce au développement de certaines zones du Maroc, cela devient plus facile et plus tentant : routes, Internet, téléphone portable, alphabétisation, concentration de richesses et de marchés, agences facilitant les transferts d'argent, etc. C'est-à-dire que les mouvements de popula- tions, qui sont les premiers vecteurs des relations économiques, s'intensifient aussi grâce aux évolutions politiques et aux infrastructures financées principalement par les États ${ }^{15}$ dans le but de développer leur pays.

De ce point de vue, l'accélération relative du développement que connaît le Maroc, loin de limiter la migration, semble plutôt la favoriser, suggérant que le lien entre développement et migration n'est pas aussi simple que le conçoivent certains décideurs. À cela s'ajoutent l'arrivée et l'installation de populations étrangères de plus en plus nombreuses, venues d'Europe et d'Afrique et utilisant le Maroc pour réaliser leurs projets personnels de mobilité. Certains utilisent ce pays comme un accélérateur de carrière, d'autres comme un relais migratoire; d'autres encore, comme un carrefour commercial.

Nous assistons bien à l'articulation entre différents régimes de mobilité et à l'émergence d'une économie de la circulation qui devient économie de la débrouille pour les plus "pauvres". En
L'accélération relative du développement que connaît le Maroc, loin de limiter la migration, semble plutôt la favoriser, suggérant alors que le lien entre développement et migration n'est pas aussi simple que le conçoivent certains décideurs. s'ouvrant dela sorte, le Maroc entre de plain-pied dans la mondialisation économique. Dans des formes très similaires, les oubliés de cette politique de développement économique essayent, souvent contre - ou en concurrence - avec l'État, eux aussi d'y entrer : pendant que l'État marocain change ses lois, développe ses infrastructures, adhère à des zones de libre échange, définit des plans d'action visant l'intégration socio-économique, culturelle et politique des Marocains à l'étranger, peaufine sa diplomatie et sa communication en matière migratoire, met en place des politiques facilitant les transferts financiers et les retours, etc., ces populations produisent une mondialisation par le bas qui intègre bien plus profondément qu'on ne le pense ce pays à l'espace euro-africain. 